\title{
Comment on Abdallah et al. entitled "Body mass index and risk of surgical site infection following spine surgery: a meta-analysis"
}

\author{
Mingbo Tian $\cdot$ Mingsheng Zhang $\cdot$ Xiaowei Guo $\cdot$ \\ Shoufeng Li
}

Received: 28 July 2013/Revised: 31 August 2013/Accepted: 31 August 2013/Published online: 12 October 2013

(C) Springer-Verlag Berlin Heidelberg 2013

The objective of this meta-analysis by Abdallah et al. [1] was to synthesize evidence from studies that evaluated the association between body mass index and risk of surgical site infection following spine surgery. It is an interesting study. Nevertheless, we have several methodological issues, which we would like to communicate with the authors.

1. Authors searched only two electronic databases (Pubmed and Embase) for studies reporting on risk of surgical site infection following spine surgery. Small number of required papers would be an important limitation of the review. We suggest at least three electronic databases should be systematically searched, such as OVID, the Cochrane Central Register, ClinicalTrials.gov and so on.

2. We suggest that the authors should evaluate the methodological quality of the selected studies, which could avoid the potential bias in the meta-analysis. Each included paper could be independently assessed by two reviewers using a standardized electronic form

\section{Tian $(\bowtie) \cdot$ S. Li}

Department of Traumatic Orthopaedics, People's Hospital of Zhengzhou, Zhengzhou 450047, Henan,

People's Republic of China

e-mail: tianmingbo450052@126.com

\section{Zhang}

Department of Orthopaedics, The Second Affiliated Hospital of Zhengzhou University, Zhengzhou 450014, Henan,

People's Republic of China

X. Guo

Department of Spinal Surgery, Zhengzhou Orthopaedics

Hospital, Zhengzhou 450052, Henan,

People's Republic of China of predefined criteria. And, we would like to know how to solve the problems if there are discrepancies between the two authors.

3. Publication language was limited to English in the meta-analysis. Therefore, the authors should mention the potential importance of language bias in the limitations of their meta-analysis.

4. The authors used a random effect model to pool the data in all the forest plots even if the $I^{2}$ value of the heterogeneity was $<50 \%$. As we know, different effect models may result in different results. Therefore, we would like to know the reason that the authors chose the random effect model for all analyses.

5. In the results part, the funnel plot was used to estimate publication bias and the symmetric inverse funnel distribution was obtained. However, it is not appropriate publication bias was assessed for adjusted OR, the number of studies was only six. As we know, a funnel plot should be inspected visually to assess for publication bias, in meta-analyses with sufficient number of studies $(>9)$.

Moreover, future studies based on prospective data collection are still needed to confirm the association between increased body mass index and spinal surgical site infection.

Conflict of interest None.

\section{Reference}

1. Abdallah DY, Jadaan MM, McCabe JP (2013) Body mass index and risk of surgical site infection following spine surgery: a metaanalysis. Eur Spine J. doi:10.1007/s00586-013-2890-6 